\title{
The Hippo effector Yorkie activates transcription by interacting with a histone methyltransferase complex through Ncoab
}

\author{
Yun Qing ${ }^{1,2 \dagger}$, Feng Yin ${ }^{1 \dagger}$, Wei Wang ${ }^{1}$, Yonggang Zheng1 ${ }^{1}$ Pengfei Guo', \\ Frederick Schozer ${ }^{3}$, Hua Deng ${ }^{1}$, Duojia Pan ${ }^{1 *}$
}

'Department of Molecular Biology and Genetics, Howard Hughes Medical Institute, Johns Hopkins University School of Medicine, Baltimore, United States; ${ }^{2}$ BCMB Graduate Program, Johns Hopkins University School of Medicine, Baltimore, United States; ${ }^{3}$ Department of Biology, Johns Hopkins University, Baltimore, United States
*For correspondence: djpan@ jhmi.edu

tThese authors contributed equally to this work

Competing interests: See page 12

Funding: See page 12

Received: 17 February 2014

Accepted: 11 July 2014

Published: 15 July 2014

Reviewing editor: Janet Rossant, University of Toronto, Canada

(c) Copyright Qing et al. This article is distributed under the terms of the Creative Commons Attribution License, which permits unrestricted use and redistribution provided that the original author and source are credited.
Abstract The Hippo signaling pathway regulates tissue growth in Drosophila through the transcriptional coactivator Yorkie (Yki). How Yki activates target gene transcription is poorly understood. Here, we identify Nuclear receptor coactivator 6 (Ncoa6), a subunit of the Trithoraxrelated (Trr) histone $\mathrm{H} 3$ lysine 4 (H3K4) methyltransferase complex, as a Yki-binding protein. Like Yki, Ncoa6 and Trr are functionally required for Hippo-mediated growth control and target gene expression. Strikingly, artificial tethering of Ncoa6 to $\mathrm{Sd}$ is sufficient to promote tissue growth and Yki target expression even in the absence of Yki, underscoring the importance of Yki-mediated recruitment of Ncoa6 in transcriptional activation. Consistent with the established role for the Trr complex in histone methylation, we show that Yki, Ncoa6, and Trr are required for normal H3K4 methylation at Hippo target genes. These findings shed light on Yki-mediated transcriptional regulation and uncover a potential link between chromatin modification and tissue growth. DOI: 10.7554/eLife.02564.001

\section{Introduction}

The Hippo signaling pathway has recently emerged as a central mechanism in organ size control, tissue regeneration, and stem cell biology (Harvey and Tapon, 2007; Badouel et al., 2009; Pan, 2010; Zhao et al., 2010; Halder and Johnson, 2011; Barry and Camargo, 2013). Initially discovered in Drosophila for its critical role in restricting imaginal disc growth, the Hippo pathway comprises several tumor suppressor proteins acting through a core kinase cascade that ultimately phosphorylates and inactivates the transcriptional coactivator Yorkie (Yki) (Huang et al., 2005). Consistent with its essential role in normal development and tissue homeostasis, YAP, the mammalian counterpart of Yki, encodes a bona fide oncogene and is overexpressed and/or activated in a wide spectrum of human cancers. Elucidating the molecular mechanism by which Yki functions as a transcriptional coactivator is not only relevant for understanding the fundamental mechanisms of growth control but also has important implications for the development of therapeutic strategies targeting the Hippo pathway in cancer and regenerative medicine.

Posttranslational modifications of histones are important features of transcriptional regulation in all eukaryotes. A particularly prevalent modification involved in transcriptional activation is histone $\mathrm{H} 3$ methylation. Drosophila contains three COMPASS (complex of proteins associated with Set1)-like histone $\mathrm{H} 3$ lysine 4 (H3K4) methyltransferase complexes, each defined by a distinct methyltransferase subunit, namely, Trithorax (Trx), Trithorax-related (Trr), and dSet1 (Mohan et al., 2011). Previous genetic 
eLife digest Cells need to work together for a multi-celled organism, such as a plant or an animal, to thrive. Many complicated signaling pathways therefore exist that allow cells to communicate with one another and to control their own activity in response to the signals that they receive. One such pathway, called the Hippo signaling pathway, regulates when cells grow and divide, which allows organs and tissues to develop correctly and helps to prevent cancerous tumors from forming.

Signaling pathways often control the activity of cells by affecting how particular genes are expressed from DNA. One way of doing this is to activate or inactivate proteins called transcription factors, which bind to sections of DNA to alter the expression of nearby genes. In fruit flies, the Hippo signaling pathway stops cells from dividing by inactivating Yorkie, a protein that binds to and activates certain transcription factors. However, exactly how Yorkie is able to activate these transcription factors was unclear.

For transcription factors to function correctly, they must be able to reach the stretch of DNA where they bind. Therefore, another way to alter gene expression is to change how the DNA is packaged in a cell. This can be done by modifying the proteins that the DNA is wrapped around, which are called histones, for example by using enzymes called methyltransferases to add methyl groups to these proteins.

Qing, Yin et al. looked at the Hippo signaling pathway in the fruit fly Drosophila, and found that the Yorkie protein only activates transcription factors when another protein called Ncoa6-which is part of a methyltransferase-binds to it. Furthermore, when the Ncoa6 protein was bound directly to the transcription factor, the tissue grew normally even when the Yorkie protein was not present. These findings reveal the importance of histone modifications in controlling tissue growth, and could provide a new direction in the search for cancer treatments.

DOI: 10.7554/eLife.02564.002

analysis has implicated Trx in the maintenance of Hox gene transcription and Trr in ecdysone receptor (EcR)-mediated gene transcription (Sedkov et al., 2003). Nuclear receptor coactivator 6 (Ncoa6) is a specific subunit of the Trr complex in both Drosophila and mammals (Mohan et al., 2011). Although its in vivo function remains undefined in Drosophila, the mammalian Ncoa6 orthologue (also known as NRC, ASC-2, TRBP, PRIP, and RAP250) is essential for embryonic development (Kuang et al., 2002; Antonson et al., 2003; Zhu et al., 2003; Mahajan et al., 2004). The mammalian Ncoa6 has been shown to potentiate the activity of nuclear hormone receptors and other DNA-binding transcription factors, at least in part, by recruiting the H3K4 methyltransferases (Mahajan and Samuels, 2008). Interestingly, like YAP, the mammalian Ncoa6 is a pro-survival and anti-apoptotic gene (Mahajan et al., 2004) and is amplified in multiple cancer types such as breast, colon, and lung cancers (Lee et al., 1999).

In this study, we identify Ncoab as a Yki-binding protein that is required for transcriptional regulation by the Hippo signaling pathway. We provide evidence showing that the transcriptional coactivator function of Yki depends on its ability to interact with $\mathrm{Ncoab}$ and that the Trr methyltransferase complex is functionally required for Hippo-mediated growth and gene expression. We further show that Yki, Ncoa6, and Trr are required for normal H3K4 methylation at Hippo target genes. Thus, Yki functions as a transcriptional coactivator, at least in part, by recruiting a H3K4 methyltransferase and altering the chromatin state of target genes.

\section{Results and discussion}

We recently reported a genome-wide RNAi screen in Drosophila S2R+ cells using a luciferase reporter driven by a minimal Hippo Responsive Element (HRE) from the Hippo target gene diap1 (Koontz et al., 2013). Briefly, Drosophila S2R+ cells were transfected with Yki- and Sd-expressing vectors, together with HRE-luciferase reporter and Pol III-Renilla expression vector as an internal control. Transfected cells were then seeded into individual dsRNA-containing 96-well plates. After RNAi depletion, HREluciferase reporter activity was measured and normalized to the Renilla control. This RNAi screen allowed us to uncover both positive and negative regulators of the HRE-luciferase reporter. We have previously characterized a negative regulator from the screen, Tgi (Koontz et al., 2013). Here, we focus on the positive transcriptional regulators (Figure 1-figure supplement1). 
One of the positive regulators identified in our primary screen is Nuclear receptor coactivator 6 (Ncoa6), which was confirmed by repeating the RNAi experiment in triplicate using re-synthesized dsRNA (Figure 1-figure supplement1). We were particularly interested in Ncoab since it contains three PPxY sequences (Figure 1A), which represent a well-established ligand binding motif for
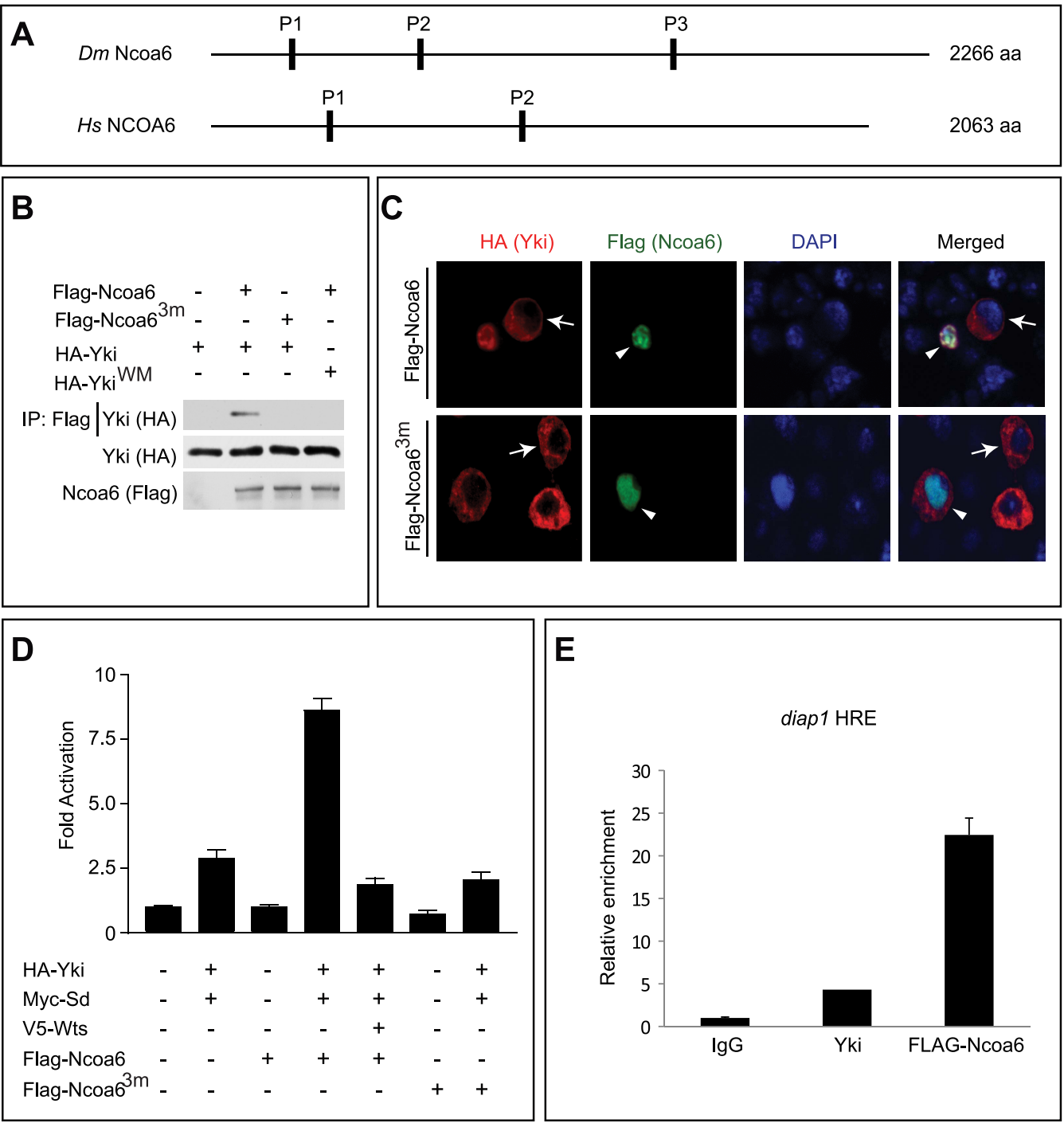

E

Figure 1. Ncoab physically interacts with Yki and regulates HRE activity. (A) Schematic protein structure of Drosophila Ncoab and its human orthologue, which contain three and two PPxY motifs, respectively. (B) S2R+ cells expressing the indicated constructs were subjected to immunoprecipitation as indicated. Note the physical interactions between Ncoab and Yki, and absence of interactions between Ncoa6 ${ }^{3 \mathrm{~m}}$ and Yki or between Ncoab and Ykimm. (C) Drosophila S2R+ cells co-transfected with HA-Yki and FLAG-Ncoab or FLAG-Ncoa6 $6^{3 m}$ constructs were stained for the indicated epitopes. Cells with or without FLAG expression are marked by arrowheads and arrows, respectively. Both FLAG-Ncoa6 and FLAG-Ncoa6 ${ }^{3 \mathrm{~m}}$ were localized to the nucleus (arrowheads), while HA-Yki was more concentrated in the cytoplasm (arrows). FLAG-Ncoab, but not FLAG-Ncoa6 ${ }^{3 m}$, induced nuclear accumulation of HA-Yki (compare arrowheads in the merged channel). (D) Luciferase activity was measured in triplicates in Drosophila S2R+ cells transfected with the indicated constructs. Ncoab, but not Ncoab ${ }^{3 m}$, enhanced Yki/Sd-mediated activation of HRE-luciferase reporter. This enhancement was suppressed by co-expression of Wts. Error bars represent standard deviations. (E) Drosophila S2R+ cells expressing FLAG-tagged Ncoab were subjected to ChIP analysis using control lgG, antibodies against FLAG or antibodies against endogenous Yki. The enrichment of HRE at the endogenous diap1 locus was measured by real-time PCR. Both Yki and FLAG-Ncoab bound to the diap1 HRE.

DOI: 10.7554/eLife.02564.003

The following figure supplement is available for figure 1:

Figure supplement1. Identification of Ncoab as a positive regulator of the HRE activity from cell-based RNAi screen. DOI: 10.7554/eLife.02564.004 
WW domains. Since Yki contains two WW domains, we hypothesized that Ncoa6 may potentiate Yki-mediated transcriptional activation through physical interactions with Yki. Indeed, epitopetagged Ncoab and Yki coimmunoprecipitated with each other in Drosophila S2R+ cells (Figure 1B). This interaction was abolished by mutating the three PPxY motifs in Ncoab (Ncoab ${ }^{3 \mathrm{~m}}$ ) or the two WW domains in Yki (YkiWM) (Figure 1B), suggesting that the Ncoab-Yki interaction was mediated by Ncoa6's PPxY motifs and Yki's WW domains. In agreement with this conclusion, we found that wild-type Ncoa6, but not $\mathrm{Ncoa}^{3 \mathrm{~m}}$, promoted nuclear accumulation of $\mathrm{Yki}$ in $\mathrm{S} 2 \mathrm{R}+$ cells in cotransfection assays (Figure 1C). Of note, a recently published Hippo pathway protein-protein interactome included Ncoab as one of 245 proteins that were co-immunopreciated by Yki (Kwon et al., 2013).

Consistent with our observation that RNAi knockdown of Ncoa6 reduced the HRE reporter activity, overexpression of Ncoab, but not the PPxY mutant Ncoa6 $6^{3 m}$, potently enhanced Yki/ Sd-mediated HRE reporter activity in Drosophila S2R+ cells (Figure 1D). In addition, the enhancement of HRE reporter activity by Ncoa6 was significantly suppressed by co-expression of the kinase Wts (Figure 1D). These results further support the importance of Ncoab-Yki interactions in Hippo-responsive transcriptional regulation. Consistent with this notion, chromatin immunoprecipitation (ChIP) revealed that Ncoab, like Yki, binds to the HRE site in the endogenous diap1 gene locus in S2R+ cells (Figure 1E).

Since mutant alleles of Ncoab are not available, we used a previously validated transgenic RNAi line (Herz et al., 2012) to assess the role of Ncoab in tissue growth and Hippo target expression in vivo. Expression of UAS-Ncoa6 RNAi by the dpp-Gal4 driver resulted in a significant decrease in the width of the dpp-expression domain in adult wings, which corresponds to the region bordered by veins $L 3$ and L4 (Figure 2A). Examination of third instar larval wing imaginal discs revealed a corresponding decrease in the expression of diap1 and four-jointed (fj), two well-characterized Hippo pathway target genes (Figure 2B-C,E-F). These results suggest that Ncoab is required for normal tissue growth and expression of Hippo target genes in vivo.

Next, we examined genetic interactions between $\mathrm{Ncoab}$ and the Hippo pathway. Overexpression of Yki or RNAi of Wts by the GMR-Gal4 driver leads to increased eye size (Figure 3D,G). Both phenotypes were suppressed by RNAi knockdown of Ncoa6 (Figure 3E,H). Conversely, knockdown of Ncoab exacerbated the small eye size induced by Sd overexpression (Figure $3 \mathrm{~J}-\mathrm{K}$ ). To further investigate the genetic interactions between Ncoab and the Hippo pathway, we used Mosaic Analysis with a Repressible Cell Marker (MARCM) (Lee and Luo, 1999) to examine the requirement of Ncoa6 in hpo mutant clones. Ncoa6 knockdown suppressed the overgrowth as well as the elevated Diap1 expression in hpo mutant clones (Figure 4A-D). In fact, hpo mutant clones with Ncoa6 knockdown showed a decrease in Diap1 expression, similar to wildtype clones with Ncoa6 knockdown (Figure 4C-D). These findings further implicate Ncoa6 in Hippo-mediated growth control and gene expression.

The physical interactions between $\mathrm{Yki}$ and Ncoab, together with the requirement for Ncoab in tissue growth and Hippo target gene expression, suggest that Yki may function as a transcriptional coactivator by interacting with Ncoab. Since Sd is the primary DNA-binding transcription partner for Yki (Koontz et al., 2013), we reasoned that fusing the DNA-binding domain of Sd with Ncoa6 may directly target Ncoab to Hippo target genes and therefore stimulate their transcription in a Ykiindependent manner (i.e., bypassing the genetic requirement for Yki). We tested this hypothesis in Drosophila wing discs using the MARCM technique. As reported before (Huang et al., 2005), yki mutant clones grew poorly and the rarely recovered clones always showed decreased Diap1 levels (Figure 5A-B). MARCM clones expressing a fusion protein between the DNA-binding domain of Sd and Ncoab (SdDB-Ncoa6) resulted in rounded clone morphology and dramatically increased Diap1 levels (Figure 5C). Significantly, the SdDB-Ncoab fusion protein, but not wild-type Ncoab, rescued the growth defect and the decreased Diap1 levels in yki mutant clone (Figure 5D-E). In fact, yki mutant clones with SdDB-Ncoab overexpression were indistinguishable in clone size and Diap1 expression compared to wild-type clones with SdDB-Ncoab overexpression (Figure 5C-D). Thus, the SdDBNcoa6 fusion protein exhibits gain-of-function activity in a Yki-independent manner. Consistent with this notion, the SdDB-Ncoab fusion protein robustly stimulated the HRE-luciferase reporter in S2R+ cells in a manner that was not suppressed by co-expression of Wts (Figure 5F), in contrast to Wts' ability to suppress the HRE-luciferase reporter activity stimulated by the co-transfection of $\mathrm{Sd}, \mathrm{Yki}$, and Ncoa6 (Figure 1D). 

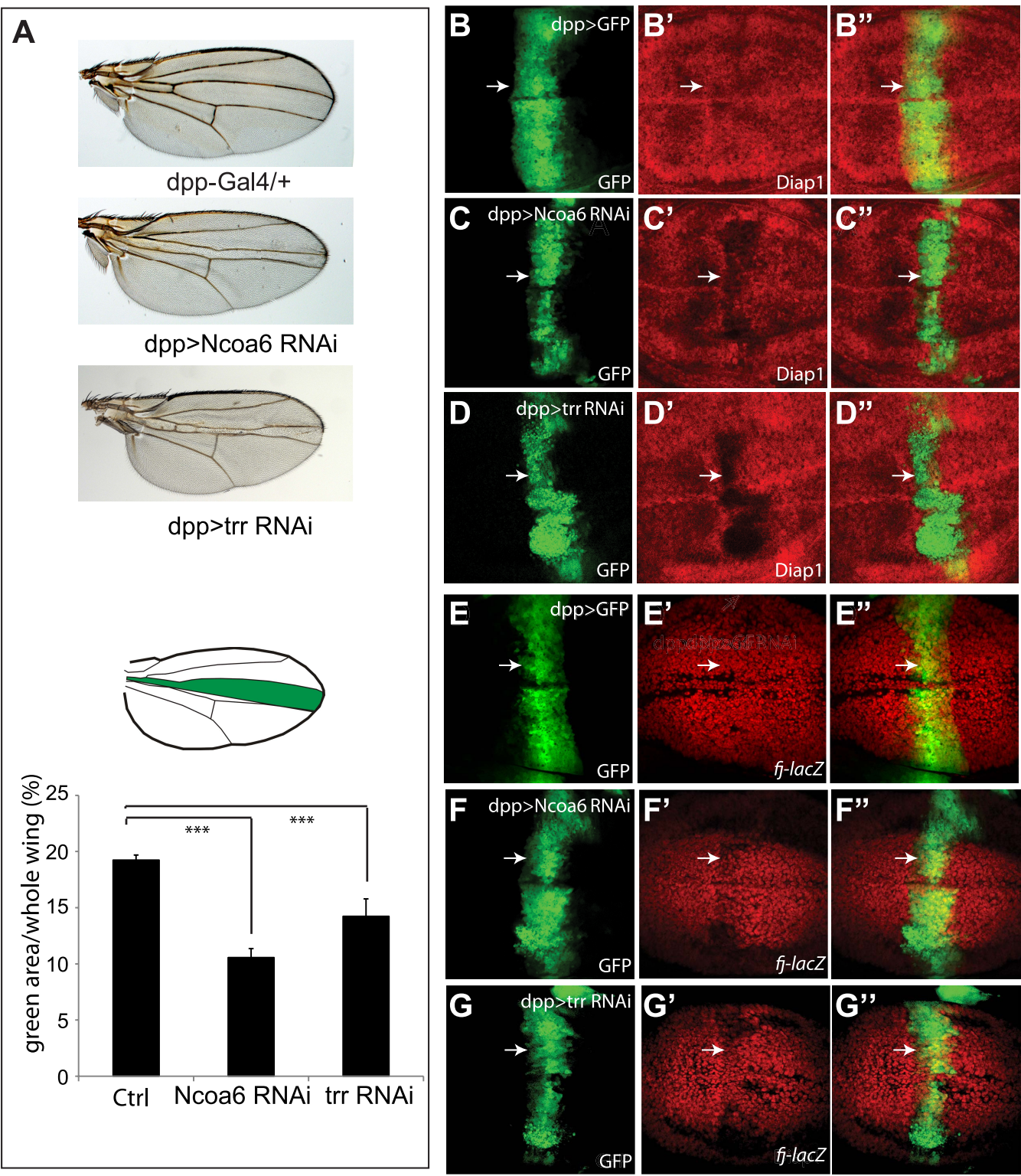

Figure 2. Ncoab and Trr are required for normal tissue growth and expression of Hippo target genes in Drosophila imaginal discs. (A) RNAi knockdown of Ncoab and Trr by dpp-Gal4 resulted in decreased area of the dpp expression domain in adult wings. The pictures were taken under the same magnification. The graph shows quantification of the dpp expression domain (green area in the schematic drawing) relative to the entire wing area (mean $\pm \mathrm{SEM}, \mathrm{n}=14,{ }^{* * *} \mathrm{p}<0.001$ ). The complete genotypes are: UAS-Dicer2; dpp-Gal4 UAS-GFP (control), UAS-Dicer2; dpp-Gal4 UAS-GFP/ UAS-Ncoa6RNAi (Ncoa6 RNAi), and UAS-Dicer2; dpp-Gal4 UAS-GFP/UAS-trrRNAi (trr RNAi). (B-G) RNAi knockdown of Ncoab or Trr resulted in decreased expression of Hippo target genes. Wing discs expressing UAS-GFP only (B and E), UAS-GFP plus Ncoab RNAi (C and F), or UAS-GFP plus trr RNAi (D and G) were stained for Diap1 (B-D) or fj-lacZ (E-G). Note the reduction of Diap1 and fj-lacZ levels upon Ncoab or Trr RNAi. The complete genotypes are: UAS-Dicer2; dpp-Gal4 UAS-GFP (B), UAS-Dicer2; dpp-Gal4 UAS-GFP/UAS-Ncoa6RNAi (C), UAS-Dicer; dpp-Gal4 UAS-GFP/UAS-trr RNAi (D), UAS-Dicer2; fj-lacZ; dpp-Gal4 UAS-GFP (E), UAS-Dicer2; fj-lacZ; dpp-Gal4 UAS-GFP/UAS-Ncoa6 RNAi (F), and UAS-Dicer2; fj-lacZ; dpp-Gal4 UAS-GFP/ UAS-trr RNAi (G).

DOI: 10.7554/eLife.02564.005

The results presented above suggest that Yki activates gene expression, at least in part, by recruiting Ncoa6. Since Ncoa6 has been reported to be a specific subunit of the Trr methyltransferase complex, we examined whether Trr, the catalytic subunit of this methyltransferase complex, is also required for Hippo-mediated growth control and gene expression. Similar to Ncoa6, expression of UAS-trr RNAi by the dpp-Gal4 driver resulted in a significant decrease in the width of the dpp-expression domain 


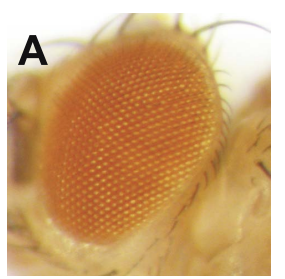

GMR-Gal4/+

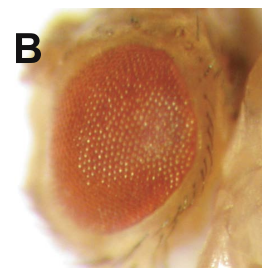

GMR $>$ Ncoa6RNAi

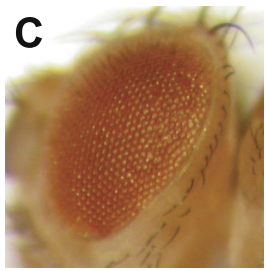

GMR>trrRNAi

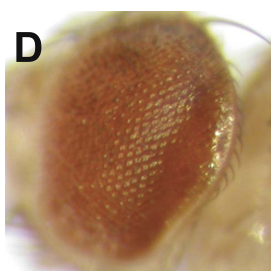

GMR>Yki

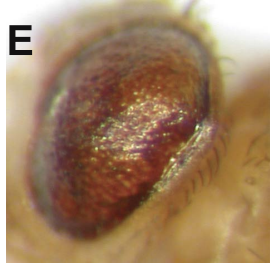

GMR $>$ Yki+Ncoa6RN

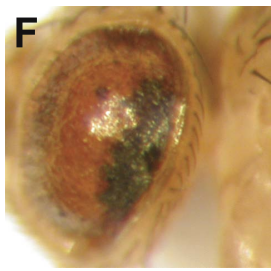

GMR>Yki+trrRNAi

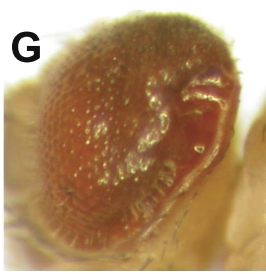

GMR $>$ WtsRNAi
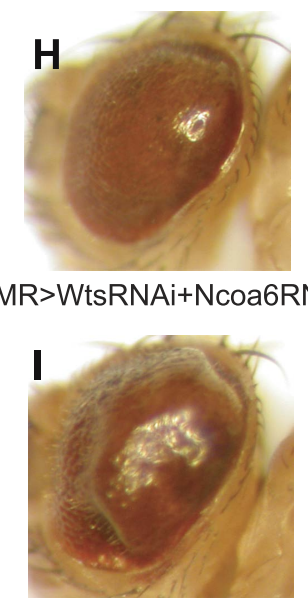

GMR $>$ WtsRNAi+trrRNAi

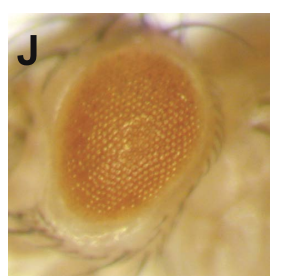

GMR $>$ Sd

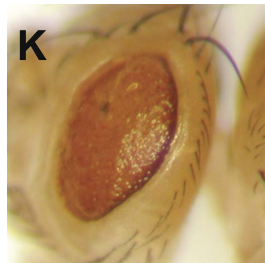

$\mathrm{GMR}>\mathrm{Sd}+\mathrm{N}$ coa6RNAi

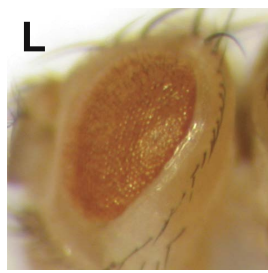

GMR>Sd+trrRNAi

Figure 3. Genetic interactions between Ncoa6-Trr and the Hippo pathway. Adult eye images of the indicated genotypes, all taken under the same magnification. (A) GMR-Gal4/+. Wild-type control. (B) GMR-Gal4/+; UASNcoab RNAi/+. RNAi knockdown of Ncoab resulted in a mild decrease in eye size (compare $\mathbf{B}$ to $\mathbf{A}$ ). (C) UASDicer2/+; GMR-Gal4/+; UAS-trr RNAi/+. RNAi knockdown of Trr resulted in no visible effects on eye size (compare C to A). (D) GMR-Gal4 UAS-Yki/+. Overexpression of Yki resulted in an increase in eye size (compare $\mathbf{D}$ to $\mathbf{A}$ ). (E) GMR-Gal4 UAS-Yki/+; UAS-Ncoa6 RNAi/+. RNAi knockdown of Ncoab suppressed eye overgrowth induced by Yki overexpression (compare $\mathbf{E}$ to $\mathbf{D}$ ). (F) UAS-Dicer2/+; GMR-Gal4 UAS-Yki/+; UAS-trr RNAi/+. RNAi knockdown of Trr suppressed eye overgrowth induced by Yki overexpression (compare $\mathbf{F}$ to D). (G) UAS-Wts RNAi/+; GMR-Gal4/+. RNAi knockdown of Wts resulted in an increase in eye size (compare $\mathbf{G}$ to $\mathbf{A}$ ). (H) UAS-Wts RNAi/+; GMR-Gal4/ UAS-Ncoa6 RNAi. RNAi knockdown of Ncoab suppressed eye overgrowth induced by Wts knockdown (compare $\mathbf{H}$ to G). (I) UAS-Dicer2/+; UAS-Wts RNAi/+; GMR-Gal4/ UAS-trr RNAi. RNAi knockdown of Trr did not obviously suppress eye overgrowth caused by Wts knockdown. (J) GMR-Gal4 UAS-Sd/+. Overexpression of Sd resulted in a decrease in eye size (compare $\mathbf{J}$ to A). (K) GMR-Gal4 UAS-Sd/+; UAS-Ncoab RNAi/+. RNAi knockdown of Ncoa6 enhanced the small eye phenotype caused by Sd overexpression (compare K to J). (L) UAS-Dicer2/+; GMR-Gal4 UAS-Sd/+; UAS-trr RNAi/+. RNAi knockdown of Trr enhanced the small eye phenotype caused by Sd overexpression (compare $\mathbf{L}$ to $\mathbf{J}$ ).

DOI: 10.7554/eLife.02564.006

in adult wings and a corresponding decrease in the Hippo target genes diap1 and fj (Figure 2A,D,G). Like Ncoa6, Trr knockdown suppressed eye overgrowth induced by Yki overexpression (Figure 3D,F) and aggravated the small eye phenotype caused by Sd overexpression (Figure $3 \mathrm{~J}, \mathrm{~L}$ ), although it did not visibly suppress eye overgrowth induced by Wts RNAi (Figure 3I). We also used MARCM to examine the requirement of Trr in hpo mutant clones. Similar to Ncoab, Trr knockdown suppressed the overgrowth as well as the elevated Diap1 expression in hpo mutant clones (Figure 4E-F). Taken together, these results implicate the Trr methyltransferase complex in Hippo-mediated growth control and target gene expression.

The Trr methyltransferase complex in Drosophila mainly affects histone H3K4 monomethylation with subtle effect on H3K4 di- or trimethylation (Herz et al., 2012; Kanda et al., 2013). To determine if Yki, Ncoa6, and Trr regulate growth in the Hippo pathway by modulating histone H3K4 methylation, we first examined the global levels of histone H3K4 methylation in Drosophila wing imaginal discs. It was reported previously that RNAi knockdown of Trr (using the en-Gal4 driver) led to a strong decrease 

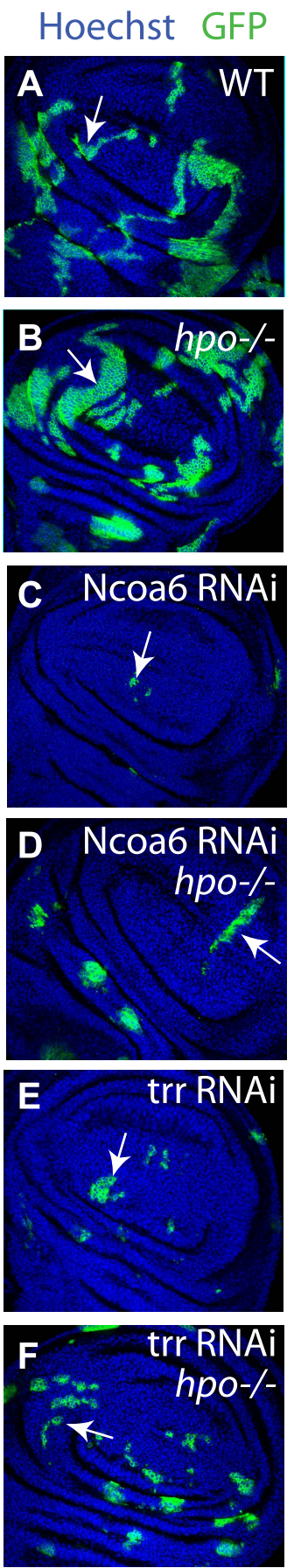
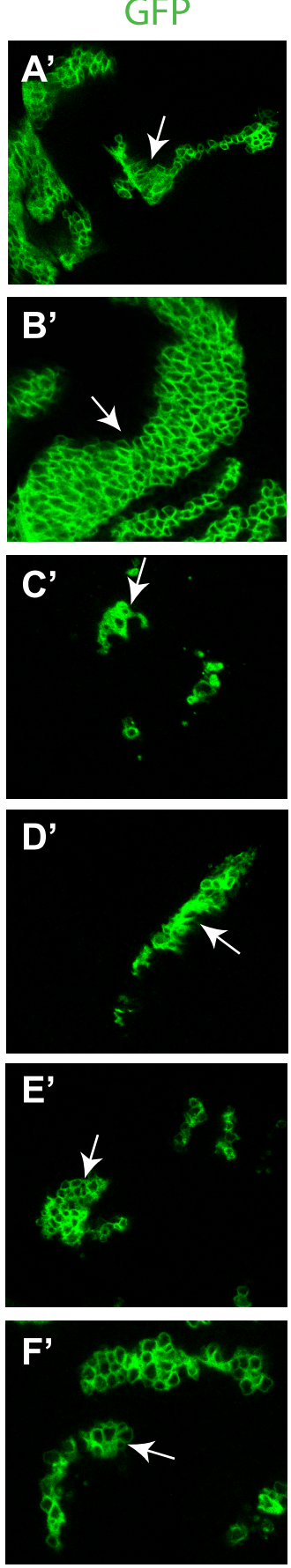

Diap1
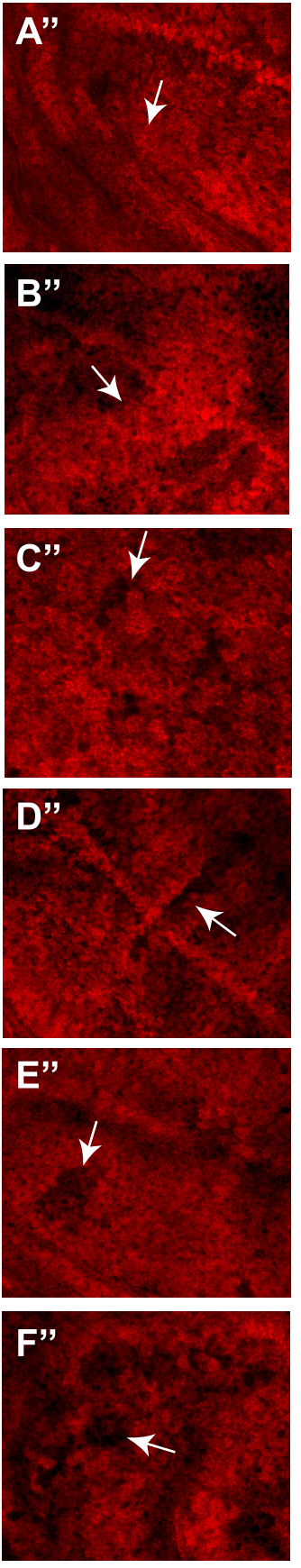

\section{GFP Diap1}
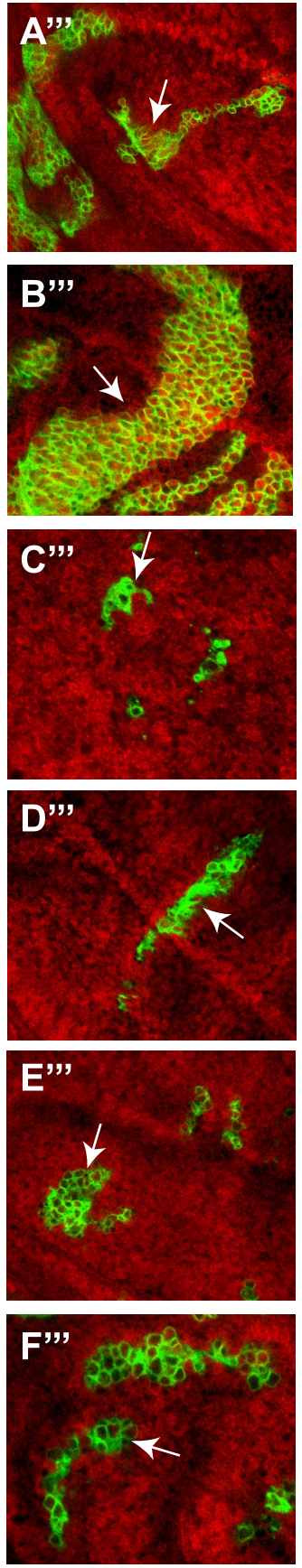

Figure 4. Ncoab and Trr are required for Hippo-mediated target gene expression. Wing discs containing GFP-marked MARCM clones were stained for Diap1 (red). For each genotype, the left most panel shows low magnification view of the wing disc (Hoechst + GFP), while the remaining three panels show higher magnification view of the same wing disc (GFP, Diap1 and GFP + Diap1). (A-F) Wing discs containing GFP-marked MARCM clones (green) of WT control (A), hpo mutant (B), Ncoa6 RNAi (C), hpo mutant with Ncoa6 RNAi (D), Trr RNAi (E), and hpo mutant with $\operatorname{Tr}$ RNAi (F). Note the increased Diap1 levels in hpo mutant clones and the decreased Diap1 levels in Ncoa6 RNAi or Trr RNAi clones. Also note the decreased Diap1 levels in hpo mutant clones with Ncoa6 RNAi or Trr RNAi.

DOI: 10.7554/eLife.02564.007 


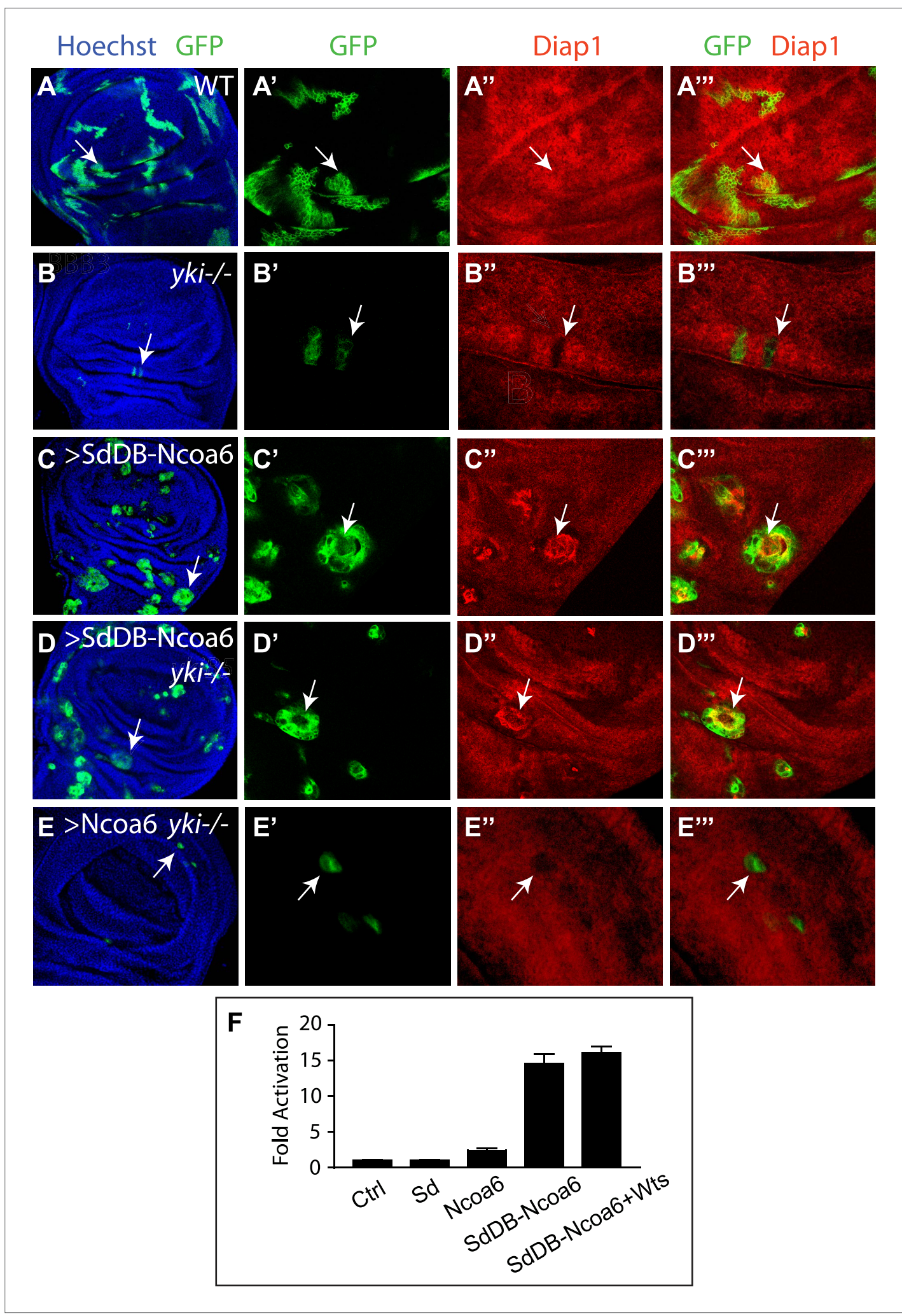

Figure 5. Fusion of Ncoab with the DNA binding domain of Sd bypasses Yki to stimulate Hippo target gene and tissue growth. (A-E) Wing discs containing GFP-marked MARCM clones (green) of WT control (A), ykiB5 (B),

SdDB-Ncoab overexpression (C), $y k^{B 5}{ }^{B 5}$ with SdDB-Ncoab overexpression (D), and $y k^{i 5}$ with Ncoab overexpression (E), were stained for Diap1 (red). For each genotype, the left most panel shows low magnification view of the wing Figure 5. Continued on next page 
Figure 5. Continued

disc (Hoechst + GFP), while the remaining three panels show higher magnification view of the same wing disc (GFP, Diap1, and GFP + Diap1). Note the decreased Diap1 expression and undergrowth of $y k^{i 5}{ }^{B 5}$ clones (B) or $y k i^{B 5} c$ lones with Ncoab overexpression (E). SdDB-Ncoab overexpression resulted in elevated Diap1 levels in $y k i^{B 5}$ clones (D). (F) Luciferase activity was measured in triplicates in Drosophila S2R+ cells transfected with the indicated constructs. Error bars represent standard deviations. Note the Wts-insensitive stimulation of the HRE-luciferase reporter by SdDB-Ncoab.

DOI: 10.7554/eLife.02564.008

in $\mathrm{H} 3 \mathrm{~K} 4 \mathrm{me} 1$ in the posterior compartment of the wing imaginal discs, while it had marginal effects on H3K4me2 and H3K4me3 levels (Mohan et al., 2011; Herz et al., 2012). It was also showed that RNAi knockdown of Ncoa6 in the posterior compartment of the wing imaginal disc resulted in a weak reduction in H3K4me1 levels (Herz et al., 2012), which we confirmed (Figure 6-figure supplement1). We further examined $\mathrm{H} 3 \mathrm{~K} 4 \mathrm{me} 2$ and $\mathrm{H} 3 \mathrm{~K} 4 \mathrm{me} 3$ levels in these imaginal discs, and observed a very subtle decrease in $\mathrm{H} 3 \mathrm{~K} 4 \mathrm{me} 3$ levels and no detectable changes in $\mathrm{H} 3 \mathrm{~K} 4 \mathrm{me} 2$ levels (Figure 6-figure supplement 1B-C). However, when we examined mutant clones of $y k i$ in the wing imaginal discs, we could not detect any changes in the global levels of H3K4me1, H3K4me2, or H3K4me3 (Figure 6-figure supplement1).

Given the negligible effect of Yki on global levels of H3K4 methylation, we investigated whether Yki modulates local H3K4 methylation on Hippo target genes. It is well established that H3K4 monomethylation marks enhancers and actively transcribed introns, while H3K4 trimethylation is enriched at active promoters and transcription start site (TSS)-proximal regions (Heintzman et al., 2007; Kharchenko et al., 2011). Interestingly, a previous genome-wide analysis in Drosophila S2 cells revealed that diap1 and ex, two well-characterized Hippo target genes, display such differential enrichment of H3K4me1 and H3K4me3 at the respective region of each gene (Herz et al., 2012) (Figure 6A). To examine the contribution of Yki, Ncoa6, and Trr to H3K4 methylation at these Hippo target genes, we knocked down each protein in S2R+ cells and performed ChIP analysis with antibodies against H3K4me1 and H3K4me3. RNAi knockdown of Yki, Ncoa6 or Trr resulted in a decrease of H3K4me3 in the TSS-proximal region of diap1 and ex (Figure 6B), which normally showed the strongest enrichment of H3K4me3 marks (Herz et al., 2012) (Figure 6A). RNAi knockdown of Yki, Ncoa6 or Trr also led to a decrease of H3K4me1 in the intronic HRE of diap1 and an upstream region of diap1 or ex which normally showed the strongest enrichment of H3K4me1 marks (Herz et al., 2012) (Figure 6C). Collectively, these data are consistent with the view that Yki activates target gene transcription by interacting with the Trr methyltransferase complex and modifying the chromatin state of the target loci.

Despite the ever expanding complexity of upstream inputs into the Hippo pathway, all of them converge on the transcriptional coactivator Yki. Thus, understanding the molecular mechanisms by which Yki regulates tissue growth and target gene expression has important implications for developmental and cancer biology. Previous studies have established that Yki functions primarily as a coactivator for Sd and that Yki promotes tissue growth by antagonizing Sd's repressor function (Wu et al., 2008; Koontz et al., 2013). Our current study has extended the previous work by identifying Ncoa6 as a Yki-binding cofactor that is required for the expression of Yki target genes. The ability of the SdDB-Ncoa6 fusion protein to rescue the growth and transcriptional defects in yki mutant clones highlights the importance of Ncoab recruitment in the transcriptional output of the Hippo pathway. Our results further suggest that Ncoa6 recruits the Trr methyltransferase complex to Hippo target genes and that Yki regulates target gene transcription by modulating local H3K4 methylation. Consistent with this view, a recent genome-wide chromatin-binding analysis revealed a correlation between Yki-bound chromatin and peaks of H3K4me3 modification in Drosophila wing discs and embryos (Oh et al., 2013). We note that, besides the H3K4 methyltransferases, the mammalian Ncoa6 has been reported to potentiate the activity of transcription factors by interacting with histone acetyltransferase CBP/p300 and several RNA binding proteins (CAPER, CoAA and PIMT) (Mahajan and Samuels, 2008). Whether these additional mechanisms also contribute to the function of Ncoa6 in Yki-mediated growth control requires further investigation. Given the biological and clinical significance of the Hippo pathway, further studies into the molecular mechanism of Ncoa6 will advance our understanding of developmental growth control and facilitate the development of novel therapeutic strategies. 

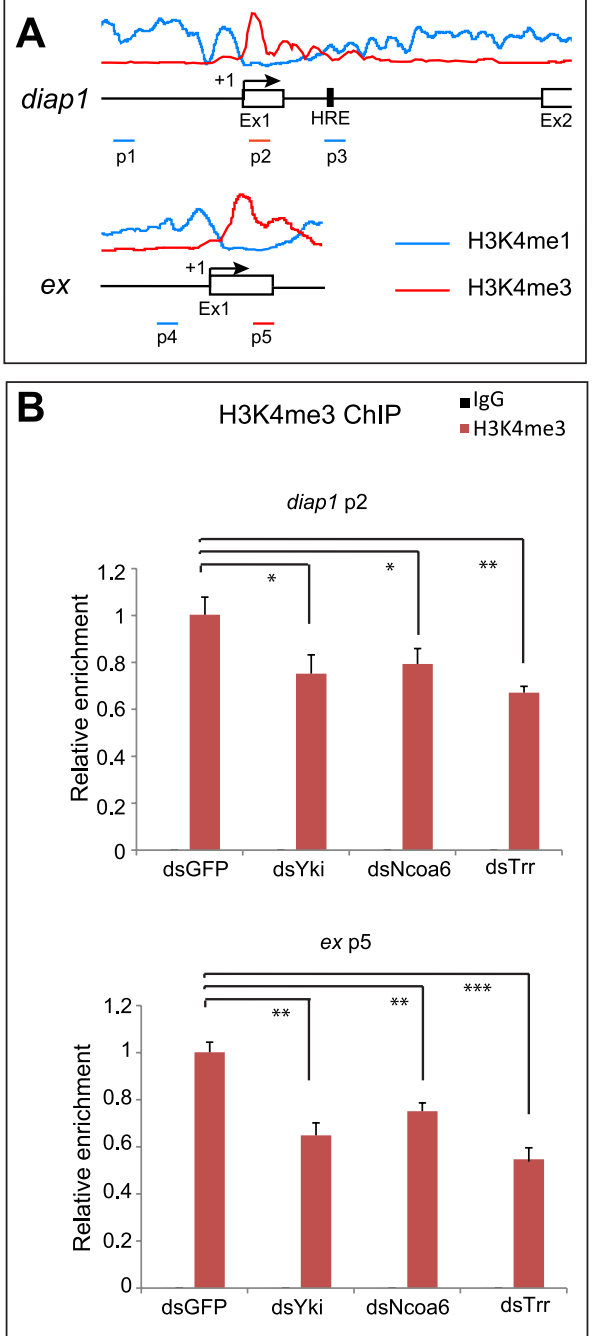
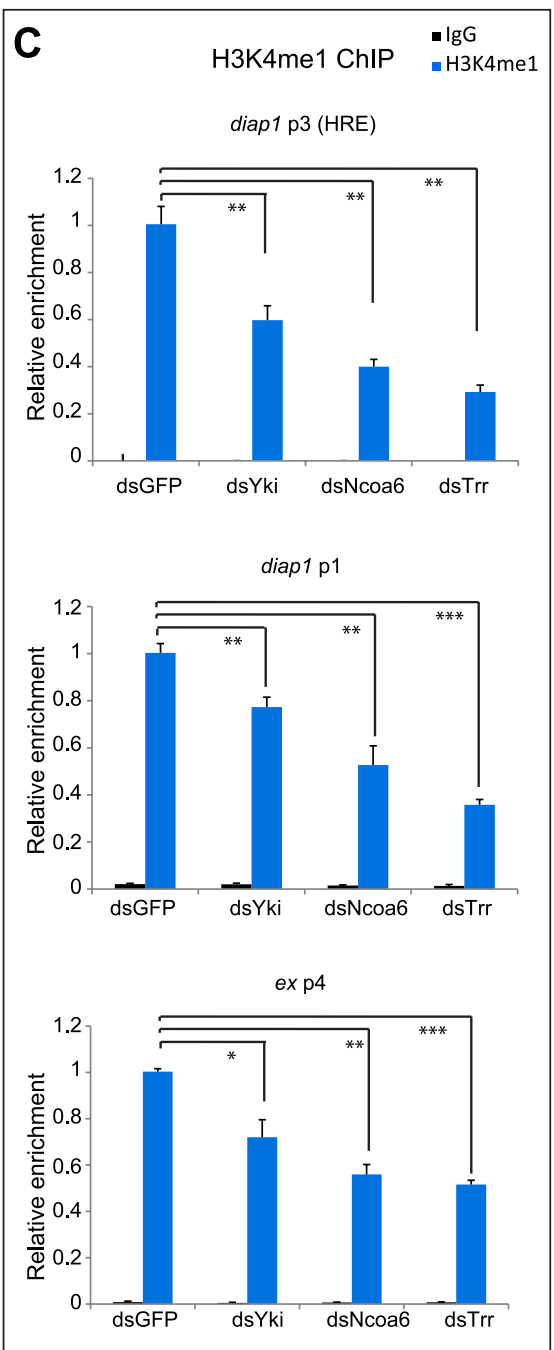

Figure 6. Yki modulates local H3K4 methylation at Hippo target genes. (A) Schematic view of diap1 and ex genomic loci analyzed by ChIP. Transcriptional start site is labeled as +1 , and p1-p5 are a series of primer sets encompassing following regions of diap1 and ex: diap1: p1: $-1951 \sim-1813, \mathrm{p} 2:+228 \sim+377, \mathrm{p} 3:+3993 \sim+4104$; ex: p4: $-749 \sim-608$, p5: +249 +393. Note that p3 covers the diap1 HRE. Also shown are the profiles of H3K4me1 (blue line) and H3K4me3 (red line) binding derived from a previously published ChIP-Seq analysis in S2 cells (Herz et al., 2012). (B and C) RNAi knockdown of Yki, Ncoab or Trr resulted in decreased H3K4me3 (B) and H3K4me1 (C) modification on Hippo target genes. ChIP analysis of $\mathrm{H} 3 \mathrm{~K} 4 \mathrm{me} 1$ or $\mathrm{H} 3 \mathrm{~K} 4 \mathrm{me} 3$ were performed in Drosophila $\mathrm{S} 2 \mathrm{R}+$ cells treated with dsRNA of GFP (control), Yki, Ncoab, or Trr. Chromatins were precipitated by control lgG or antibodies against $\mathrm{H} 3 \mathrm{~K} 4 \mathrm{me} 1$ and $\mathrm{H} 3 \mathrm{~K} 4 \mathrm{me}$. The enrichment of ChIP products on diap and ex was measured by real-time PCR using the indicated primers. ${ }^{* \star *} p<0.001,{ }^{\star \star} p<0.01,{ }^{*} p<0.05$.

DOI: 10.7554/eLife.02564.009

The following figure supplement is available for figure 6:

Figure supplement1. Ncoab, but not Yki, regulates global levels of H3K4 methylation.

DOI: 10.7554/eLife.02564.010

\section{Materials and methods}

\section{Molecular cloning and mutagenesis}

A full-length Ncoa6 cDNA corresponding to the BDGP annotated RD transcript was generated from mRNA of Drosophila third instar larvae, using SuperScript(R) III One-Step RT-PCR System with Platinum Taq High Fidelity (Life Technologies, Carlsbad, California). Mutations of PPxY motifs were generated 
in Ncoab using the QuikChange II XL Site-Directed Mutagenesis Kit (Agilent, Santa Clara, CA), replacing tyrosine $(\mathrm{Y}$ ) with alanine (A). To generate Sd-Ncoab, Sd DNA binding domain was inserted to the $\mathrm{N}$-terminal of Ncoab. FLAG-tag was inserted to the N-terminal of Ncoab, Ncoab ${ }^{3 m}$, and Sd-Ncoab and cloned into the attB-UAS vector.

\section{Drosophila genetics}

Flies with the following genotypes have been described previously: $y k i{ }^{B 5}$, UAS-Yki (Huang et al., 2005), hpo ${ }^{42-48}$ (Wu et al., 2003), fj-lacZ reporter fj-II (Villano and Katz, 1995), UAS-Sd (Halder et al., 1998), UAS-Wts RNAi (Stock ID VDRC v106174). The UAS-Ncoa6 RNAi and UAS-trr RNAi lines have been validated previously (Herz et alo, 2012) and were obtained from Bloomington Drosophila Stock Center (Stock ID 34964 and 29,563). attB-UAS-Ncoa6 and attB-UAS-FLAG-SdDB-Ncoa6 transgenes were inserted into the $86 \mathrm{Fa}$ attP acceptor site by phiC31-mediated site-specific transformation (Bischof et al., 2007).

For the MARCM experiments in Figure 4, the following clones were induced 48-60 hr after egg deposition and heat shocked at $37^{\circ} \mathrm{C}$ for $15 \mathrm{~min}$ :

UAS-GFP hs-FLP; FRT42D, Tub-Gal80/FRT42D; Tub-Gal4/+

UAS-GFP hs-FLP; FRT42D, Tub-Gal80/FRT42D hpo42-48; Tub-Gal4/+

UAS-GFP hs-FLP; FRT42D, Tub-Gal80/FRT42D; Tub-Gal4/UAS-Ncoa6RNAi

UAS-GFP hs-FLP; FRT42D, Tub-Gal80/FRT42D hpo 42-48; Tub-Gal4/UAS-Ncoa6RNAi

UAS-Dicer2/UAS-GFP hs-FLP; FRT42D, Tub-Gal80/FRT42D; Tub-Gal4/UAS-trrRNAi

UAS-Dicer2/UAS-GFP hs-FLP; FRT42D, Tub-Gal80/FRT42D hpo ${ }^{42-48 ; ~ T u b-G a l 4 / U A S-t r r R N A i ~}$

For the MARCM experiments in Figure 5A-E, the following clones were induced $72-84 \mathrm{hr}$ after egg deposition and heat shocked at $37^{\circ} \mathrm{C}$ for $10 \mathrm{~min}$ :

UAS-GFP hs-FLP; FRT42D, Tub-Gal80/FRT42D; Tub-Gal4/+

UAS-GFP hs-FLP; FRT42D, Tub-Gal80/FRT42D yki ${ }^{B 5}$; Tub-Gal4/+

UAS-GFP hs-FLP; FRT42D, Tub-Gal80/FRT42D; Tub-Gal4/UAS-SdDB-Ncoa6

UAS-GFP hs-FLP; FRT42D, Tub-Gal80/FRT42D yki ${ }^{35}$; Tub-Gal4/UAS-SdDB-Ncoa6

UAS-GFP hs-FLP; FRT42D, Tub-Gal80/FRT42D yki ${ }^{\text {B5; }}$ Tub-Gal4/UAS-Ncoa6

\section{Drosophila cell culture, transfection, immunoprecipitation, immunoflurescence, and luciferase reporter assay}

Drosophila S2R+ cells were cultured in Schneider's Drosophila Medium (Life Technologies) supplemented with $10 \%$ fetal bovine serum and antibiotics. HA-Yki and HA-YkiWM have been described previously (Huang et al., 2005). Luciferase assay was carried out using Dual Luciferase Assay System (Promega, Madison, WI) and a FLUOstar Luminometer (BMG LabTechnologies, Germany). Tansfection, immunopreciptation, and immunofluorescence staining of S2R+ cells were performed using standard protocols as described (Yin et al., 2013).

\section{ChIP assays}

ChIP assays were performed according to a previously described protocol (Wang et al., 2009). Briefly, $\sim 5 \times 10^{6}$ (for ChIP assay with histone methylation antibodies) or $1.5 \times 10^{7}$ (for CHIP assay with Yki or FLAG antibodies) S2R + cells were cross-linked with $1 \%$ formaldehyde and sonicated to an average fragment size between $200 \mathrm{bp}$ and $500 \mathrm{bp}$. Two micrograms of control lgG or specific antibodies, including rabbit a-H3K4me1 (8895, Abcam, England) and rabbit a-H3K4me3 (8580, Abcam), and $50 \mu$ lof protein $\mathrm{G}$ agarose were used in each ChIP assay. The immunoprecipitated DNA was quantified using real-time PCR. All values were normalized to the input. The primers for analyzing the ChIP NA are provided as follows:
p1 Forward: TGTTCTTGTTGGTGCTGCTT
p1 Reverse: TTAATGCTGGCATGGTTTCA
p2 Forward: TAAAACTGGGGCTCACCTTG
p2 Reverse: TCGTGTTCACGGAAAATCAA
p3 (HRE) Forward: ACGAACACGAAGACCAAA
p3 (HRE) Reverse: CTCCAAGCCAGTTTGATT
p4 Forward: AAAAGAGGGAAGAGGGAGCA 
p4 Reverse: GAATCGGAATCGGAACTTGA

p5 Forward: TCGCACTCGCCTCAATTAC

p5 Reverse: CAGCACCAACTTTTCGGAGT

\section{Acknowledgements}

We thank Jianzhong Yu, Melissa Jones and Elizabeth Garcia for technical assistance. This study was supported in part by grants from the National Institutes of Health (EY015708). DP is an investigator of the Howard Hughes Medical Institute.

\section{Additional information}

\begin{tabular}{|c|c|c|}
\hline unding & & \\
\hline Funder & Grant reference number & Author \\
\hline $\begin{array}{l}\text { Howard Hughes Medical } \\
\text { Institute }\end{array}$ & & $\begin{array}{l}\text { Yun Qing, Feng Yin, Wei Wang, } \\
\text { Yonggang Zheng, Pengfei Guo, } \\
\text { Frederick Schozer, Hua Deng, } \\
\text { Duojia Pan }\end{array}$ \\
\hline $\begin{array}{l}\text { National Institutes of } \\
\text { Health }\end{array}$ & NIH EY015708 & $\begin{array}{l}\text { Yun Qing, Feng Yin, Wei Wang, } \\
\text { Yonggang Zheng, Pengfei Guo, } \\
\text { Frederick Schozer, Hua Deng, } \\
\text { Duojia Pan }\end{array}$ \\
\hline
\end{tabular}

The funders had no role in study design, data collection and interpretation, or the decision to submit the work for publication.

Author contributions

YQ, FY, Conception and design, Acquisition of data, Analysis and interpretation of data, Drafting or revising the article; WW, YZ, Conception and design, Acquisition of data, Analysis and interpretation of data; PG, HD, Acquisition of data; FS, Acquisition of data, Analysis and interpretation of data; DP, Conception and design, Analysis and interpretation of data, Drafting or revising the article

\section{References}

Antonson P, Schuster GU, Wang L, Rozell B, Holter E, Flodby P, Treuter E, Holmgren L, Gustafsson JA. 2003. Inactivation of the nuclear receptor coactivator RAP250 in mice results in placental vascular dysfunction. Molecular and Cellular Biology 23:1260-1268. doi: 10.1128/MCB.23.4.1260-1268.2003.

Badouel C, Garg A, McNeill H. 2009. Herding Hippos: regulating growth in flies and man. Current Opinion in Cell Biology 21:837-843. doi: 10.1016/j.ceb.2009.09.010.

Barry ER, Camargo FD. 2013. The Hippo superhighway: signaling crossroads converging on the Hippo/Yap pathway in stem cells and development. Current Opinion in Cell Biology 25:247-253. doi: 10.1016/j. ceb.2012.12.006

Bischof J, Maeda RK, Hediger M, Karch F, Basler K. 2007. An optimized transgenesis system for Drosophila using germ-line-specific phiC31 integrases. Proceedings of the National Academy of Sciences of the United States of America 104:3312-3317. doi: 10.1073/pnas.0611511104.

Halder G, Johnson RL. 2011. Hippo signaling: growth control and beyond. Development 138:9-22. doi: 10.1242/ dev.045500.

Halder G, Polaczyk P, Kraus ME, Hudson A, Kim J, Laughon A, Carroll S. 1998. The vestigial and scalloped proteins act together to directly regulate wing-specific gene expression in Drosophila. Genes \& Development 12:3900-3909. doi: 10.1101/gad.12.24.3900.

Harvey K, Tapon N. 2007. The Salvador-Warts-Hippo pathway-an emerging tumour-suppressor network. Nature Reviews Cancer 7:182-191. doi: 10.1038/nrc2070.

Heintzman ND, Stuart RK, Hon G, Fu Y, Ching CW, Hawkins RD, Barrera LO, Van CS, Qu C, Ching KA, Wang W, Weng Z, Green RD, Crawford GE, Ren B. 2007. Distinct and predictive chromatin signatures of transcriptional promoters and enhancers in the human genome. Nature Genetics 39:311-318. doi: 10.1038/ng1966.

Herz HM, Mohan M, Garruss AS, Liang K, Takahashi YH, Mickey K, Voets O, Verrijzer CP, Shilatifard A. 2012. Enhancer-associated H3K4 monomethylation by Trithorax-related, the Drosophila homolog of mammalian MII3/ Mll4. Genes \& Development 26:2604-2620. doi: 10.1101/gad.201327.112. 
Huang J, Wu S, Barrera J, Matthews K, Pan D. 2005. The Hippo signaling pathway coordinately regulates cell proliferation and apoptosis by inactivating Yorkie, the Drosophila homolog of YAP. Cell 122:421-434. doi: 10.1016/j.cell.2005.06.007.

Kanda H, Nguyen A, Chen L, Okano H, Hariharan IK. 2013. The Drosophila ortholog of MLL3 and MLL4, trithorax related, functions as a negative regulator of tissue growth. Molecular and Cellular Biology 33:1702-1710. doi: 10.1128/MCB.01585-12.

Kharchenko PV, Alekseyenko AA, Schwartz YB, Minoda A, Riddle NC, Ernst J, Sabo PJ, Larschan E, Gorchakov AA, Gu T, Linder-Basso D, Plachetka A, Shanower G, Tolstorukov MY, Luquette LJ, Xi R, Jung YL, Park RW, Bishop EP, Canfield TK, Sandstrom R, Thurman RE, MacAlpine DM, Stamatoyannopoulos JA, Kellis M, Elgin SC, Kuroda MI, Pirrotta V, Karpen GH, Park PJ. 2011. Comprehensive analysis of the chromatin landscape in Drosophila melanogaster. Nature 471:480-485. doi: 10.1038/nature09725.

Koontz LM, Liu-Chittenden Y, Yin F, Zheng Y, Yu J, Huang B, Chen Q, Wu S, Pan D. 2013. The hippo effector yorkie controls normal tissue growth by antagonizing scalloped-mediated default repression. Developmental Cell 25:388-401. doi: 10.1016/j.devcel.2013.04.021.

Kuang SQ, Liao L, Zhang H, Pereira FA, Yuan Y, DeMayo FJ, Ko L, Xu J. 2002. Deletion of the cancer-amplified coactivator AIB3 results in defective placentation and embryonic lethality. The Journal of Biological Chemistry 277:45356-45360. doi: 10.1074/jbc.C200509200.

Kwon Y, Vinayagam A, Sun X, Dephoure N, Gygi SP, Hong P, Perrimon N. 2013. The Hippo signaling pathway interactome. Science 342:737-740. doi: 10.1126/science.1243971.

Lee T, Luo L. 1999. Mosaic analysis with a repressible cell marker for studies of gene function in neuronal morphogenesis. Neuron 22:451-461. doi: 10.1016/S0896-6273(00)80701-1.

Lee SK, Anzick SL, Choi JE, Bubendorf L, Guan XY, Jung YK, Kallioniemi OP, Kononen J, Trent JM, Azorsa D, Jhun BH, Cheong JH, Lee YC, Meltzer PS, Lee JW. 1999. A nuclear factor, ASC-2, as a cancer-amplified transcriptional coactivator essential for ligand-dependent transactivation by nuclear receptors in vivo. The Journal of Biological Chemistry 274:34283-34293. doi: 10.1074/jbc.274.48.34283.

Mahajan MA, Das S, Zhu H, Tomic-Canic M, Samuels HH. 2004. The nuclear hormone receptor coactivator NRC is a pleiotropic modulator affecting growth, development, apoptosis, reproduction, and wound repair. Molecular and Cellular Biology 24:4994-5004. doi: 10.1128/MCB.24.11.4994-5004.2004.

Mahajan MA, Samuels HH. 2008. Nuclear receptor coactivator/coregulator NCoA6(NRC) is a pleiotropic coregulator involved in transcription, cell survival, growth and development. Nuclear Receptor Signaling 6:e002. doi: 10.1621/nrs.06002.

Mohan M, Herz HM, Smith ER, Zhang Y, Jackson J, Washburn MP, Florens L, Eissenberg JC, Shilatifard A. 2011. The COMPASS family of H3K4 methylases in Drosophila. Molecular and Cellular Biology 31:4310-4318. doi: 10.1128/MCB.06092-11.

Oh H, Slattery M, Ma L, Crofts A, White KP, Mann RS, Irvine KD. 2013. Genome-wide association of Yorkie with chromatin and chromatin-remodeling complexes. Cell Reports 3:309-318. doi: 10.1016/j.celrep.2013.01.008.

Pan D. 2010. The hippo signaling pathway in development and cancer. Developmental Cell 19:491-505. doi: 10.1016/j.devcel.2010.09.011.

Sedkov Y, Cho E, Petruk S, Cherbas L, Smith ST, Jones RS, Cherbas P, Canaani E, Jaynes JB, Mazo A. 2003. Methylation at lysine 4 of histone H3 in ecdysone-dependent development of Drosophila. Nature 426:78-83. doi: 10.1038/nature02080.

Villano JL, Katz FN. 1995. Four-jointed is required for intermediate growth in the proximal-distal axis in Drosophila. Development 121:2767-2777.

Wang W, Huang L, Huang Y, Yin JW, Berk AJ, Friedman JM, Wang G. 2009. Mediator MED23 links insulin signaling to the adipogenesis transcription cascade. Developmental Cell 16:764-771. doi: 10.1016/j. devcel.2009.04.006.

Wu S, Huang J, Dong J, Pan D. 2003. hippo encodes a Ste-20 family protein kinase that restricts cell proliferation and promotes apoptosis in conjunction with salvador and warts. Cell 114:445-456. doi: 10.1016/ S0092-8674(03)00549-X.

Wu S, Liu Y, Zheng Y, Dong J, Pan D. 2008. The TEAD/TEF family protein Scalloped mediates transcriptional output of the Hippo growth-regulatory pathway. Developmental Cell 14:388-398. doi: 10.1016/j. devcel.2008.01.007.

Yin F, Yu J, Zheng Y, Chen Q, Zhang N, Pan D. 2013. Spatial organization of hippo signaling at the plasma membrane mediated by the tumor suppressor Merlin/NF2. Cell 154:1342-1355. doi: 10.1016/j. cell.2013.08.025.

Zhao B, Li L, Lei Q, Guan KL. 2010. The Hippo-YAP pathway in organ size control and tumorigenesis: an updated version. Genes \& Development 24:862-874. doi: 10.1101/gad.1909210.

Zhu YJ, Crawford SE, Stellmach V, Dwivedi RS, Rao MS, Gonzalez FJ, Qi C, Reddy JK. 2003. Coactivator PRIP, the peroxisome proliferator-activated receptor-interacting protein, is a modulator of placental, cardiac, hepatic, and embryonic development. The Journal of Biological Chemistry 278:1986-1990. doi: 10.1074/jbc.C200634200. 